\title{
Fabrication of High-Aspect-Ratio Cylindrical Micro-Structures Based on Electroactive Ionogel/Gold Nanocomposite
}

\author{
Edoardo Milana ${ }^{1,+} \oplus$, Tommaso Santaniello ${ }^{2, *}{ }^{\dagger}$, Paolo Azzini ${ }^{2}$, Lorenzo Migliorini ${ }^{2}$ \\ and Paolo Milani ${ }^{2}$ (D) \\ 1 Department of Mechanical Engineering, KU Leuven and Flanders Make, Celestijnenlaan 300, 3001 Leuven, \\ Belgium; edoardo.milana@kuleuven.be \\ 2 CIMaINa, Department of Physics, Università degli Studi di Milano, Via Celoria 16, 20133 Milano, Italy; \\ paolo.azzini@studenti.unimi.it (P.A.); lorenzo.migliorini@unimi.it (L.M.); paolo.milani@mi.infn.it (P.M.) \\ * Correspondence: tommaso.santaniello@unimi.it \\ + The authors equally contributed to the work.
}

Received: 29 September 2020; Accepted: 21 October 2020; Published: 26 October 2020

\begin{abstract}
We present a fabrication process to realize 3D high-aspect-ratio cylindrical micro-structures of soft ionogel/gold nanocomposites by combining replica molding and Supersonic Cluster Beam Deposition (SCBD). Cylinders' metallic masters $(0.5 \mathrm{~mm}$ in diameter) are used to fabricate polydimethylsiloxane (PDMS) molds, where the ionogel is casted and UV cured. The replicated ionogel cylinders (aspect ratio > 20) are subsequently metallized through SCBD to integrate nanostructured gold electrodes (150 nm thick) into the polymer. Nanocomposite thin films are characterized in terms of electrochemical properties, exhibiting large double layer capacitance $\left(24 \mu \mathrm{F} / \mathrm{cm}^{2}\right)$ and suitable ionic conductivity $(0.05 \mathrm{mS} / \mathrm{cm})$ for charge transport across the network. Preliminary actuation tests show that the nanocomposite is able to respond to low intensity electric fields (applied voltage from $2.5 \mathrm{~V}$ to $5 \mathrm{~V}$ ), with potential applications for the development of artificial smart micro-structures with motility behavior inspired by that of natural ciliate systems.
\end{abstract}

Keywords: polymer/metal nanocomposites; electroactive actuators; soft robotics

\section{Introduction}

Advances in nanotechnology and materials science are fueling new research paths in many engineering fields. A bursting trend is soft robotics, where the boundary between materials and devices is increasingly blurring in an attempt at mimicking biological systems [1]. New soft materials and microfabrication techniques enable devices that have been traditionally a prerogative of stiff components, such as actuators, sensors and even structural elements. In this sense, there is a need to create soft active matter, where materials can mechanically respond to external stimuli or vice versa. Many soft materials have been developed to deform in the presence of magnetic, fluidic, thermal, chemical and electrical stimuli [2]. Among those, electro-active polymers (EAPs) materials deform when subjected to an electric field. EAPs are normally divided in two sub-categories: dielectric and ionic materials, according to their deformation mechanism [3]. In ionic materials, the deformation is caused by a differential swelling of the polymer induced by the migration of ions in response to an applied electric field [4,5]. Ionic Polymer/Metal Composites (IPMCs) harness this mechanism when a voltage difference is applied between two electrodes embedded in the ionic polymer. Traditionally, the ionic polymer is Nafion or Flemion, whose surfaces are plated or coated with gold or platinum [6]. However, those materials have a relatively high Young modulus and traditional metallization techniques are not suitable for the production of stable, compliant and well-adherent electrodes $[7,8]$. 
Recently, we have developed a new type of IPMC material using ionogels and cluster-assembled nanostructured electrodes produced by Supersonic Cluster Beam Deposition (SCBD) to realize soft bending actuators $[9,10]$. This metallization approach relies on the use of supersonic beams seeded with neutral metal nanoparticles that are directed towards the ionogel target at room temperature. SCBD enables the high-throughput fabrication of thin conductive electrodes, highly resilient against deformation, without significantly altering the mechanical properties of the polymer [10]. However, those actuators are limited by their planar shape, whereas different morphologies would allow more complex deformation patterns and optimize the response according to the application. Soft artificial microswimmers, for example, inspired by the movement of biological cilia and flagella, have better hydrodynamic performances when designed with cylindrical shapes [11].

Here, we report on a new fabrication process of cylindrical ionogel/gold nanocomposites by combining replica molding and SCBD to produce 3D micro-actuators with high-aspect-ratio. The base material is a hydrophilic soft co-polymer that is able to incorporate large amounts of an imidazolium-based ionic liquid. After SCBD fabrication, the nanocomposite exhibited large electrochemical capacitance, suitable ionic conductivity and electromechanical response under the application of low intensity electrical stimuli (from $2.5 \mathrm{~V}$ to $5 \mathrm{~V}$ ). The proposed manufacturing strategy has potential to be employed for the realization of smart electroactive structures for bio-inspired soft micro-components fabrication.

\section{Materials and Methods}

\subsection{Ionogel Synthesis and Thin Films Fabrication}

The ionogel matrix is a co-polymer based on 2-hydroxyethylmethacrylate (HEMA) and acrylonitrile (AN), chemically cross-linked using polyethylene glycole diacrylate (PEGDA, $500 \mathrm{Mw}$ ) under UV light irradiation. The polymer formulation was engineered to favor the incorporation of an imidazolium-based ionic liquid into the network, 1-(2-hydroxyethyl)-3-methylimidazolium tetrafluoroborate (OHMIM) [12]. This compound provides the presence of free ions in the material, which are able to migrate across the polymeric matrix under the application of an electric field. The polymer synthesis took place at room temperature in ambient conditions by dissolving the monomers and cross-linker into the ionic liquid. The ratio between the monomers was kept as HEMA: $\mathrm{AN}=1.6 \mathrm{w} / \mathrm{w}$, while the cross-linker amount was $0.02 v / w$ with respect to the total monomers' weight. The ratio between the monomers and ionic liquid was fixed at $1.24 w / w$. After mixing the reagents into a $4 \mathrm{~mL}$ test tube (total mixture volume per sample was $1.5 \mathrm{~mL}$ ), a $15 \mathrm{mg} / \mu \mathrm{L}$ solution of the photo-initiator (2,2-Dimethoxy-2-phenylacetophenone) in dimethyl sulfoxide solution was added into the pre-polymer (30 $\mu \mathrm{L}$ per stock solution). The relative amount of reagents was chosen on an empirical basis in order to obtain a soft elastic gel with elastomeric-like mechanical properties after UV photo-crosslinking at $365 \mathrm{~nm}$. More specifically, the amount of monomers was calibrated in order to provide the gel with hydroxyl groups, present in the HEMA monomer, to favor the interaction with the OHMIM and to simultaneously toughen the matrix with the AN monomer. The balance between the ionic liquid phase and polymeric matrix confers the overall macroscopic softness and elasticity to the material. The latter was tuned and enhanced by the introduction of a high molecular weight cross-linker in the structure.

We used a simple molding approach to produce thin films of the prepared ionogel (namely, poly(HEMA-co-AN)/OHMIM). The fabrication consisted in pouring the pre-polymer solution into a spacer $(0.5 \mathrm{~mm}$ thick) enclosed between two microscope glass slides and by exposing the system to UV light (light source was an UVP BlackRay, $100 \mathrm{~W}$ ) for $60 \mathrm{~min}$. These films served as benchmark reference samples to assess the electrical, electrochemical and electromechanical properties of the material after SCBD metallization. 


\subsection{Replica Molding}

We employed replica molding to fabricate high-aspect ratio $(>20)$ cylindrical ionogel-based micro-structures. This approach relied on the use of commercial syringe needles ( $0.5 \mathrm{~mm}$ diameter $)$ as masters to generate a negative mold in polydimethylsiloxane (PDMS). PDMS is an elastomer with high chemical and thermal stability, widely employed for the molding of microfluidic devices and soft robotic components due to its ability to accurately replicate structures down to fractions of microns [13]. We chose metal needles as template because of their suitable size and shape, ease of demolding from the cured elastomer and for their easy purchase availability. The replica molding process to produce ionogel-based micro-components is depicted in Figure 1.

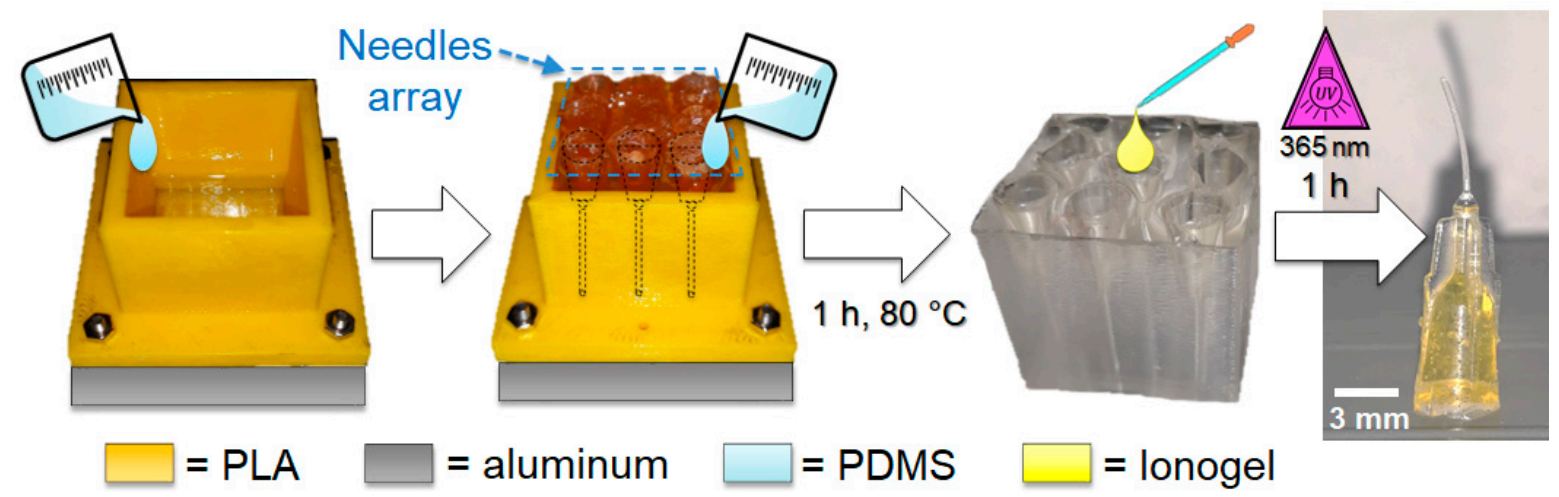

Figure 1. Schematic of the replica molding process to produce ionogel-based cylindrical micro-structures.

A $3 \times 3$ array of needles was positioned inside a dedicated box $(28 \mathrm{~mm} \times 28 \mathrm{~mm}$ wide, $30 \mathrm{~mm}$ high) fabricated by means of fused filament fabrication (FFF) 3D printing using poly lactic acid (PLA) and a Delta 2040 machine from WASP (Massa Lombarda (RA), Italy). The box was fastened to an aluminum basis that was covered with a first layer of PDMS (around $1 \mathrm{~mm}$ thick, elastomer: curing agent ratio $=10: 1 w / w)$ where the needle tips were fixed. The box was then filled by casting PDMS (prepared with the same elastomer to curing agent ratio) in between the needles and up to the free surface. After polymerization in an oven at $80^{\circ} \mathrm{C}$ for one hour, the new master mold was obtained by removing the needles from the polymer and unfastening the box. The negative replicas of the needles were then filled with the ionogel pre-polymer solution using a pipette and the mold was exposed to UV light at $365 \mathrm{~nm}$ for two hours. After complete cross-linking, cylindrical ionogels were demolded and dried in a vacuum overnight prior to further processing and testing.

\subsection{Supersonic Cluster Beam Deposition for Ionogel/Gold Nanocomposites Fabrication}

Supersonic Cluster Beam Deposition (SCBD) is a well-established metallization technique extensively employed for the fabrication of polymer/metal nanocomposites with controlled electrical and functional properties [10,14-16]. SCBD relies on the use of highly collimated supersonic beams seeded with neutral metal nanoparticles directed onto a target substrate to fabricate nanostructured metallic films with thickness in the range of hundreds of nm (Figure 2). The details on the operational principle are reported elsewhere [17] and summarized here below. A pulsed flux of inert gas is injected inside the cluster source at high pressure (40 bar), where a rotating metallic rod is subjected to high-voltage electric discharges $(700 \mathrm{~V})$. The atoms sputtered from the target metal then condense in neutral clusters. Due to a pressure gradient, the nanoparticles are carried by the gas expansion into a second chamber, forming a cluster-seeded supersonic beam [18]. The beam then passes through a skimmer and reaches the deposition chamber, where clusters impinge onto the target substrate. A quartz microbalance is used to monitor the amount of deposited material in real time. The thickness of the metal layer is solely dependent on the number of clusters deposited, which is in turn determined by the deposition time [17]. This process parameter simultaneously controls the resulting surface 
roughness of the film at the nanoscale, with typical values ranging from several nanometers to a few tens of nanometers $[19,20]$.

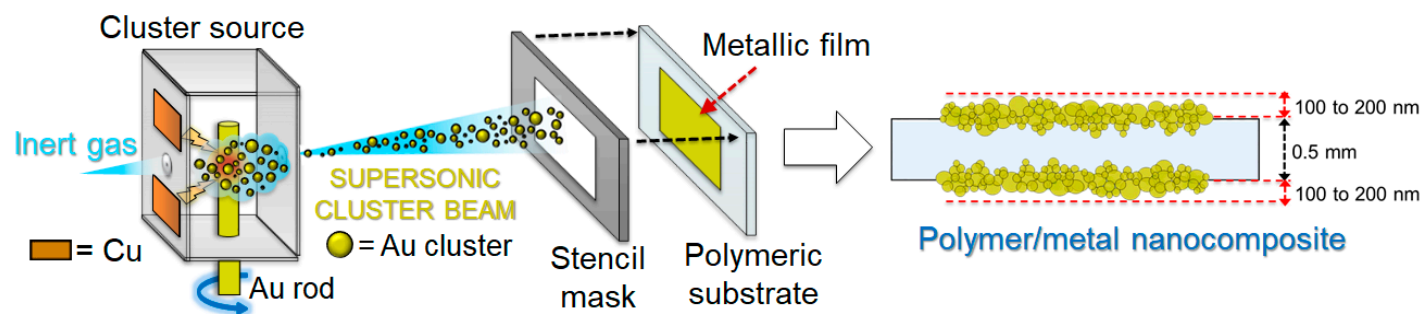

Figure 2. Simplified schematic of the SCDB process for polymer/metal nanocomposites fabrication.

Due to the low kinetic energy (around $0.5 \mathrm{ev} / \mathrm{atom}$ ), they do not undergo significant deformation or fragmentation upon impact with the target [17]. When using soft polymers as deposition substrates, the metallic film partially interpenetrates with the polymeric network, resulting in the formation of a polymer/metal nanocomposite, with no damage or carbonization of the polymer [14]. When dealing with the metallization of ionic electroactive polymers, these features are particularly advantageous. In fact, the generation of a cluster-assembled metallic layer inside the polymeric matrix enhances the ionic charge accumulation efficiency under the application of an electric field at the electrodes, due to the available large surface area and porosity of the metal phase [9]. As compared to other classical metal deposition techniques, such as atomic layer deposition [21,22], SCBD enables a physical interlocking of the metallic structure to the polymer rather than the fabrication of a surface thin film. Moreover, it does not require surface functionalization of the substrate or the presence of precursor molecules and reactive surface groups on the target to favor the deposition process. It also avoids the triggering of chemical reactions into the polymers upon fabrication and takes place at room temperature. The SCBD process to fabricate polymer/metal nanocomposites is schematized in Figure 2.

We used this approach to fabricate conductive gold electrodes with large surface area on ionogel-based thin films and cylindrical micro-structures. SCBD was performed using stencil masks in both cases to define the geometry of the electrodes on the opposite sides of the samples, obtaining a classical capacitor-like geometry. The equivalent thickness of the fabricated layer (i.e., the thickness of a thin film produced on a rigid flat substrate using the same amount of clusters) was around $150 \mathrm{~nm}$. The amount of gold deposited on the ionogel was measured in-situ using the dedicated quartz micro-balance. The equivalent thickness of the composite was assessed off-line using a profilometer (KLA Tencor, Milpitas, CA, USA), measuring the thickness of a cluster-assembled film deposited on a reference silicon substrate, mounted beside the target ionogel during deposition.

For planar ionogel films, the electrodes' surface area was defined by an $8 \mathrm{~mm} \times 8 \mathrm{~mm}$ squared region using hollow aluminum masks (produced through milling). For the metallization of ionogel-based cylindrical structures, we designed and fabricated, by means of stereolithograpic 3D printing, a sample support able to fit the ionogels, as represented in Figure 3. The printer employed was a Form 2 from FormLabs, while the material was a standard FormLabs Clear Resin. With this approach, the electrodes fabricated on the cylindrical micro-structures were $0.4 \mathrm{~mm}$ wide metallic strips covering the whole length of the needle replica, avoiding electrical contact between the metallized regions of the nanocomposite. The ionogels were fully metallized using rastering, with the objective of connecting the larger basis underlying the cylinders (i.e., the ionogel replica of the plastic connector for syringe fitting) with external electronic equipment to prompt the actuation of the micro-structure. The sheet resistance of both nanocomposite cylinders and thin films was assessed using a multimeter. 


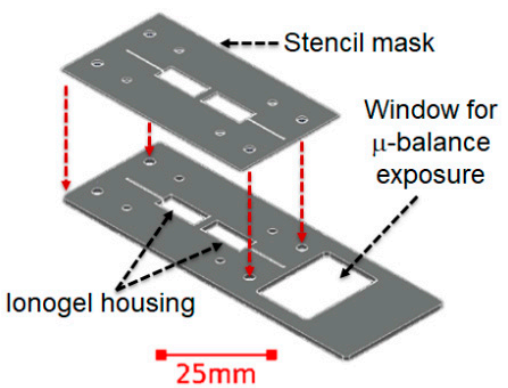

(a)

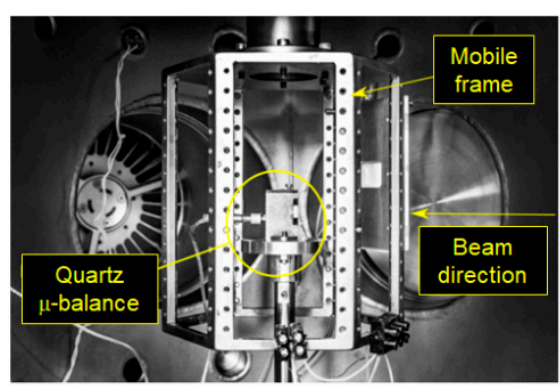

(b)

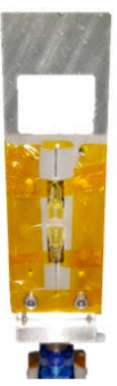

(c)

Figure 3. (a) Computer-Aided Design (CAD) drawing of the sample support used for the fabrication of cylindrical ionogel/metal nanocomposites; (b) photograph of the deposition chamber where samples are mounted on a mobile holder for metallization. The holder can rotate and translate in the zenithal direction during deposition using a programmable remote controller; (c) photograph of the assembled support system for cylindrical ionogel/metal nanocomposite fabrication.

\subsection{Electrochemical and Electromechanical Characterization}

The electrochemical properties of the nanocomposites were tested using electrochemical impedance spectroscopy (EIS) and cyclic voltammetry (CV) on the thin film samples. These techniques provide relevant information on the charge accumulation ability and resistive behavior of the nanocomposites, as well as on their electrochemical stability [23]. EIS was conducted by applying a sinusoidal voltage signal to the nanocomposite and by recording the corresponding current response. We kept the voltage amplitude at $5 \mathrm{mV}$, while the frequency was varied between $10^{6} \mathrm{~Hz}$ and $10^{-2} \mathrm{~Hz}$. The system impedance is determined by the phase shift between the input and output signals and current amplitude [16]. CVs were carried out by applying a voltage ramp varying between $-5 \mathrm{~V}$ and $5 \mathrm{~V}$, with a scan rate of $10 \mathrm{mV} / \mathrm{s}$, and by recording the induced current. A typical voltammogram displays the presence of symmetric current peaks located at the voltage values where redox reactions take place at the electrodes. The equipment used for these tests was a Gamry Reference 600 potentiostat.

The actuation behavior of the samples was assessed using a dedicated setup consisting of a waveform generator and a power supply, connected to a custom-designed control circuit, to apply electrical sinusoidal signals of different frequencies $(0.1 \mathrm{~Hz}$ to $10 \mathrm{~Hz})$ and amplitudes $(1 \mathrm{~V}$ to $5 \mathrm{~V})$ to the nanocomposites. We used thin metal plates ( $0.2 \mathrm{~mm}$ thick) as interconnects, employing a custom-designed FFF printed support to regulate the clamping pressure of the electrical contact to the actuators. The plates were made of commercial copper-based layers coated in-house with a thin gold film (200 nm thick, produced by means of physical vapor deposition) to provide electrical connections with suitable electrical and electrochemical stability in the voltage range explored [12]. The linear displacement of the benders was registered in real time using a camera, and the acquired images were then processed by means of a MATLAB script developed in-house. This analysis tool permits tracking of the motion of the actuator tip along the deformation path using the Object Tracking algorithm of the MATLAB Computer Vision Toolbox.

\section{Results and Discussions}

\subsection{Ionogel Molding and Nanocomposite Fabrication}

The replication technique was very effective in reproducing the ionogel cylindric samples with good dimensional accuracy. Samples dimensions were observed and compared to the masters using transmission and reflection optical microscopy. The average diameter of the ionogel cylinders was $485 \mu \mathrm{m}$, whereas the needles' diameter was measured as $515 \mu \mathrm{m}$ (both values calculated over ten measurements conducted along the cylinder) with a percentage standard deviation of roughly $1 \%$. This size discrepancy between the metal master and ionogel diameter is fairly acceptable, considering the 
drying process that the polymers underwent. We analyzed the moisture-sensitivity character of the ionogel by measuring the weight of thin film samples straight after synthesis and every two hours under exposure to ambient conditions $\left(\mathrm{RH}=45 \%, \mathrm{~T}=23^{\circ} \mathrm{C}\right)$. We then calculated the weight increase with respect to the initial weight, which was systematically found to be $3.5 \%$ (measurements were conducted on five samples). To the best of our knowledge, there is no report in the literature on fabrication approaches enabling the production of high-aspect-ratio cylindrical electroactive structures based on soft polymers at this length scale in 3D.

For ionogel/gold nanocomposites fabrication, we decided to use an equivalent thickness of the deposited metal equal to $150 \mathrm{~nm}$ in order to provide the ionogels with well-adherent conductive electrodes for efficient electrical signal transmission. In general, the increase in the electrical conductivity of a polymer during SCBD is observed in correspondence to a critical thickness of the forming metallic film $[9,14,24]$. At this transition point (the electrical percolation threshold), clusters start to form a sufficiently high number of physical connections to permit the flow of an electrical current in the system [24]. As the number of clusters increases, the film tends to behave like an ohmic conductor [14,24]. At this stage, a further increase in the layer thickness does not affect the electrical conductivity or the electrochemical performance of the composite material $[9,14,24]$.

After SCBD metallization, the nanocomposites showed sheet resistance with values ranging from 50 to $100 \mathrm{Ohm}^{*} \mathrm{~cm}$ for the thin films and between 300 and $500 \mathrm{Ohm}$ for the $10 \mathrm{~mm}$ long cylinder. Images of both thin films and cylindrical ionogel/gold nanocomposites are reported in Figure 4. This resistance increase can be attributed to the poor surface finishing of the metal masters, used as purchased without further polishing, which is reproduced on the ionogel surface after replica molding [25].

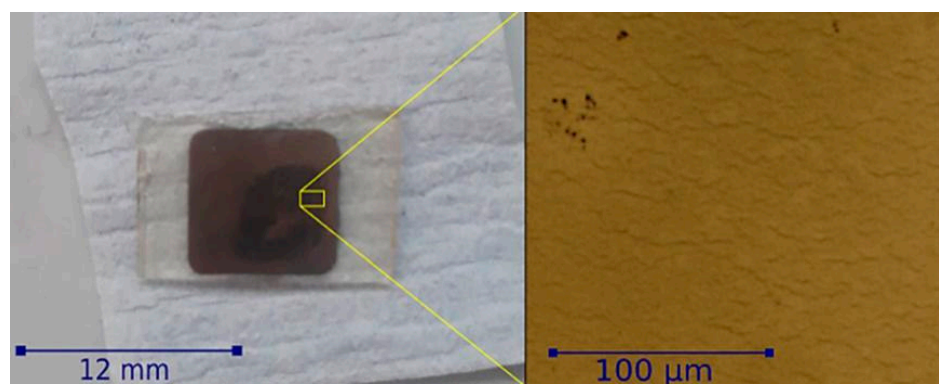

(a)

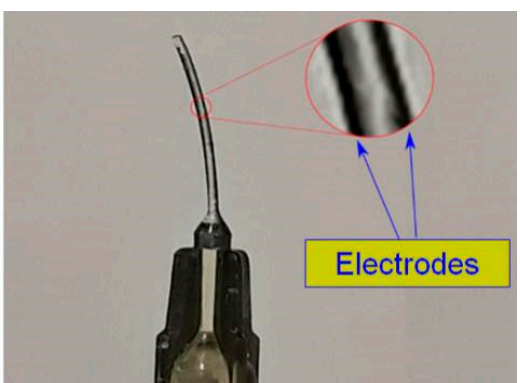

(b)

Figure 4. (a) Photograph and microscope images of an ionogel/gold nanocomposite film and (b) photograph of a nanocomposite cylindrical micro-actuator. Inset shows a magnification of the cylinder, highlighting the physical separation of the electrodes fabricated on the opposite sides of the structure.

We observed no electrical contact between the metal electrodes fabricated on the opposite sides of the cylinders (Figure $4 \mathrm{~b}$ ). This suggests that the stencil mask and sample support employed for SCBD metallization were suitable for the correct electrode patterning of the ionogel. However, the electrodes deposited on the cylindrical body were also shown to be electrically disconnected from the larger basis underlying the micro-actuator. This electrical discontinuity is probably due to the presence of abrupt cross-sectional changes along the ionogel replica in the direction parallel to the beam. These geometrical variations implied a physical gap in the deposited metal film located at the interface between the $0.5 \mathrm{~mm}$ diameter cylinder and the rest of the nanocomposite structure. This issue could be circumvented by employing custom-designed master molds with a smoother profile (e.g., by means of 3D printing VAT photopolymerization) for ionogel fabrication and by tilting the sample holder in the deposition chamber to favour the metallization of non-planar structures. This last solution has already proven to be successful for SCBD fabrication of 3D freeform polymer/metal nanocomposites [26,27]. 
SCBD is a high-throughput technique that can be employed for the scale-up manufacturing of soft actuators, as previously reported $[9,10]$. The substrate rastering in the direction perpendicular to the cluster beam can be exploited for the metallization of large areas [14], delivering gold clusters only on the substrate. It relies on the ablation of small metal precursors [17], with small amounts (around $1 \mathrm{mg} / 500 \mathrm{~mm}^{2}$ ) and no significant waste of raw material.

\subsection{Ionogel/Gold Nanocomposites Electrochemical and Actuation Properties}

Results of the electrochemical analysis conducted on the nanocomposites are reported in Figure 5. In Figure 5a, it is possible to observe the Bode plot correlating the impedance modulus and phase with the frequency of the input voltage signal. A resistive behaviour was registered at intermediate frequencies where the phase value is close to $0^{\circ}$, while, in the low frequency domain, the presence of a double-layer capacitance was observed, with a maximum phase shift of about $-60^{\circ}$ at $10^{-2} \mathrm{~Hz}$. By modelling the nanocomposite samples as simple RC circuits [9], we calculated the values of the specific capacitance and ionic conductivity, which was equal to $24 \mu \mathrm{F} / \mathrm{cm}^{2}$ and $0.05 \mathrm{mS} / \mathrm{cm}$, respectively.

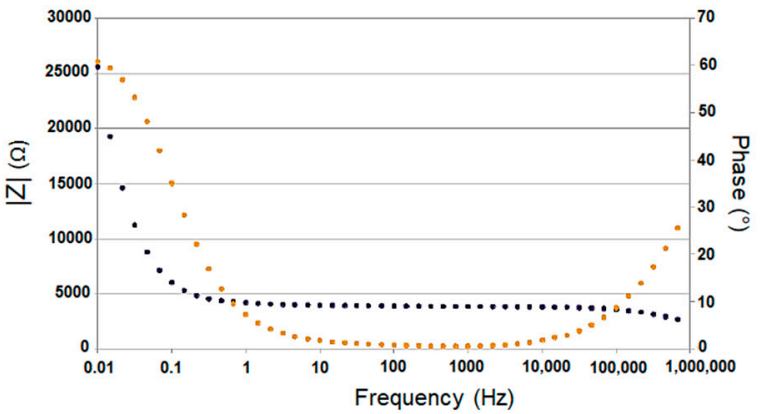

(a)

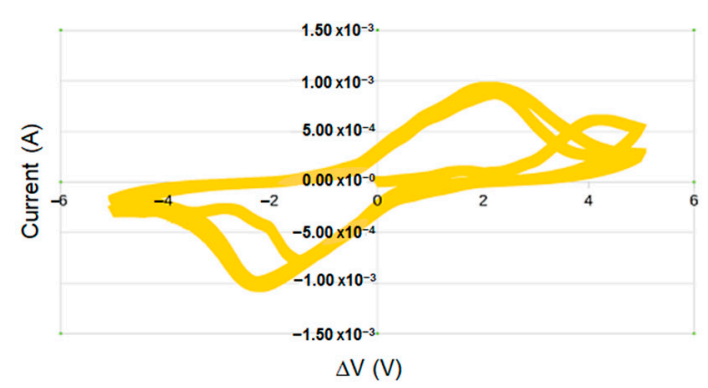

(b)

Figure 5. Electrochemical analysis of the ionogel/gold nanocomposites: (a) Bode plot and (b) cyclic voltammograms registered with the potentiostat.

The value of the electrochemical capacitance is in line with that reported in the literature for this class of nanocomposites (several tens to a few hundreds of $\mu \mathrm{F} / \mathrm{cm}^{2}[10,16]$ ), for which the large surface area of the cluster-assembled electrodes have a determinant role in favouring an efficient ionic charge accumulation. On the other hand, the ionic conductivity was moderately low when compared to other ionogel/metal nanocomposites produced by means of SCBD (typical values between $0.1 \mathrm{mS} / \mathrm{cm}$ and $1 \mathrm{mS} / \mathrm{cm}[10,16])$, but still suitable to guarantee the migration of the charge carriers inside the polymeric matrix under the application of an electric field.

This behaviour can be associated with the lack of ionic groups (and relative solvated mobile counter-ions) in the polymer and with a significant interaction between the ionic liquid and the network backbone, damping the overall ionic mobility [28,29]. The voltammograms (Figure 5b) show the presence of symmetric current peaks in the $\mathrm{mA}$ order that can be ascribed to the activation of redox reactions. The electrochemical window of stability of the nanocomposites is between $-2.5 \mathrm{~V}$ and $2.5 \mathrm{~V}$.

Electromechanical actuation tests were first performed on $15 \mathrm{~mm}$ long and $1 \mathrm{~mm}$ wide nanocomposite strips ( $0.5 \mathrm{~mm}$ thick), manually cut from the fabricated thin films. The actuators underwent electrical stimulation in a cantilever configuration, clamped to a $3 \mathrm{~mm} \times 3 \mathrm{~mm}$ wide support. Material reactivity to the application of electric fields started at $2.5 \mathrm{~V}$. Before testing the composite frequency response, a $5 \mathrm{~V}$ continuous voltage was applied to the actuators to assess the maximum linear displacement of the tip, which was $0.3 \mathrm{~mm}$. This limited motion can be ascribed to the combination of the ionogel softness and actuator thickness, the latter being higher with respect to the typical thickness $(0.07$ to $0.3 \mathrm{~mm})$ of the ionic actuators described in the literature $[5,7,30]$. The percentage strain $(\varepsilon)$ of the nanocomposites was calculated to have a more direct comparison with IPMCs actuators of different geometries [10]. More specifically, $\varepsilon$ was computed as $\varepsilon=2 \mathrm{~d} \times \mathrm{t} /\left(\mathrm{L}^{2}+\mathrm{d}^{2}\right)$, where $\mathrm{d}$ is the measured 
linear tip displacement, $\mathrm{t}$ is the actuator thickness and $\mathrm{L}$ the cantilever free length. The nanocomposite $\varepsilon$ value was $0.1 \%$, which is compatible with the most recent results reported in the literature concerning soft ionic electroactive actuators [31,32].

Frequency tests were then carried out on the samples. The tip displacement tracking was conducted by applying sinusoidal voltage signals with a fixed amplitude of $3 \mathrm{~V}$ and $5 \mathrm{~V}$. A graph showing the registered tip displacement over time at three different frequencies at $5 \mathrm{~V}$ is reported in Figure $6 \mathrm{a}$. We could observe deformation of the actuators in phase with the applied electrical signal up to $10 \mathrm{~Hz}$, with displacements decreasing as the frequency was increased. The actuation was shown to be stable and repeatable over time in the frequency range explored. Cylindrical nanocomposite micro-structures could not be tested in the same setup used for the cantilevers, due to the abovementioned electrical disconnection between the cylinder and the underlying interconnection basis and to criticalities encountered in the attempt to electrically connect the soft cylinder with the actuation control circuit. Therefore, we used remote electrodes, consisting of $10 \mathrm{~cm} \times 10 \mathrm{~cm}$ wide stainless steel plates, to apply an electric field to cylindrical ionogel samples located between the electrodes (Figure 6b).

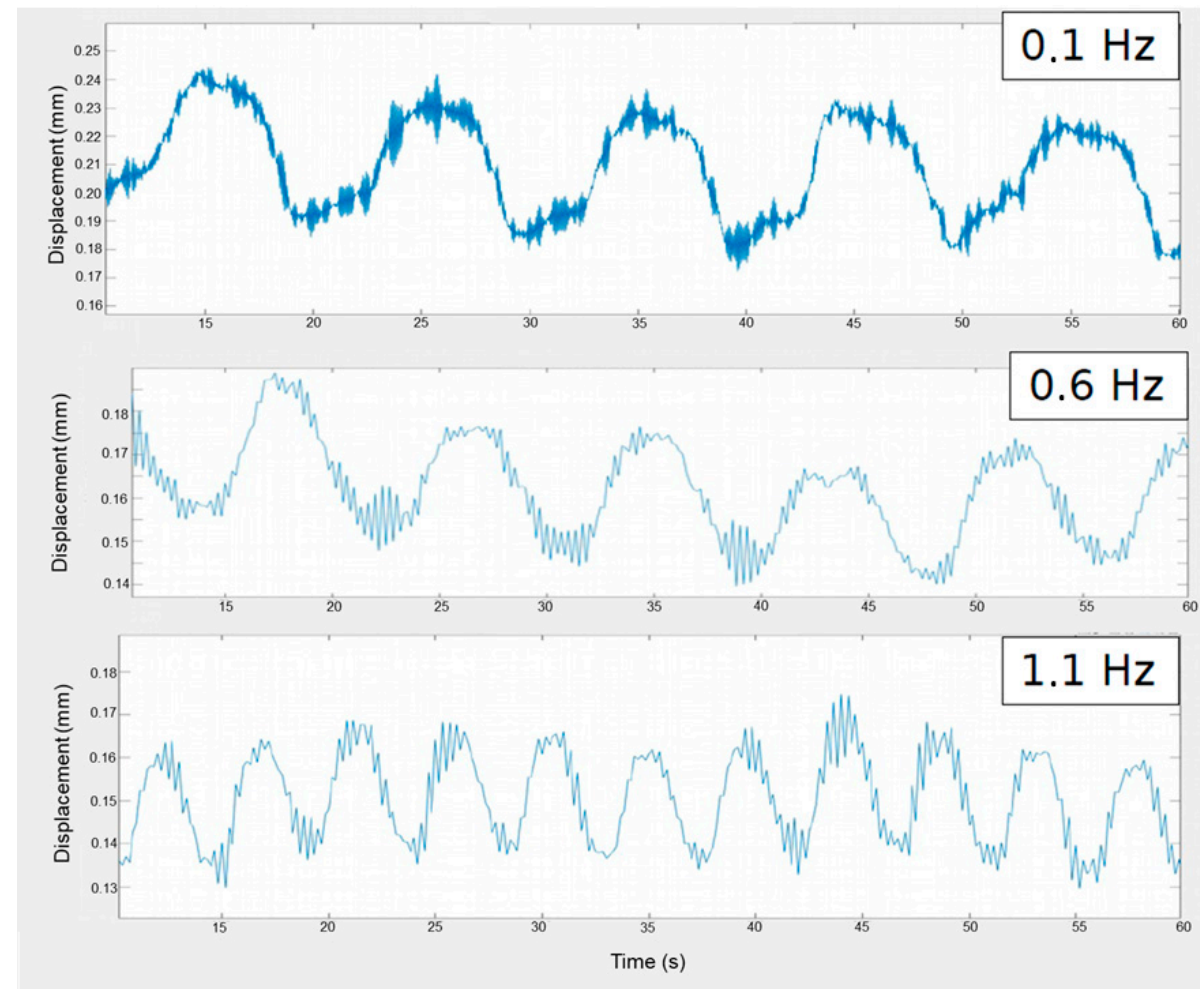

(a)

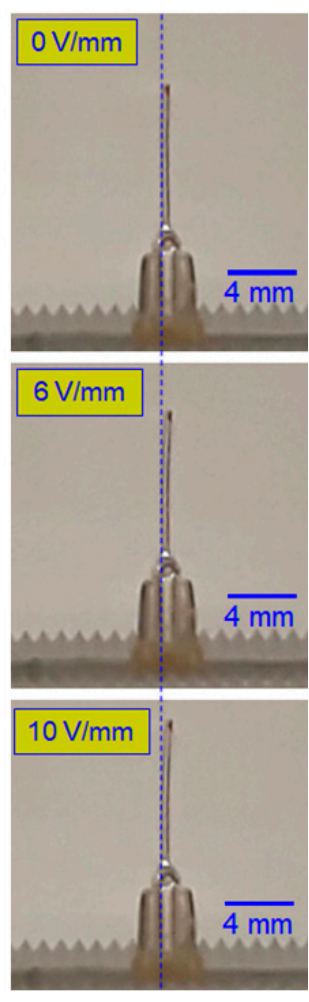

(b)

Figure 6. (a) Linear displacement of the cantilever nanocomposite actuator at $5 \mathrm{~V}$ for three frequency values. (b) Snapshots of the bending of an ionogel cylinder triggered using remote electrodes to apply the electrical stimulus.

The relative metal plate distance was $60 \mathrm{~mm}$. To obtain electric field values comparable to that employed to trigger the actuation of the nanocomposite thin films (between $6 \mathrm{~V} / \mathrm{mm}$ and $10 \mathrm{~V} / \mathrm{mm}$ ), we employed a high-voltage generator operating in the $\mathrm{kV}$ range. The obtained tip displacements for the cylinders were systematically lower, but comparable to that of the thin films (Figure 6b).

Although the charge accumulation mechanism in the case of remote electrodes differs from that taking place at metallic structures integrated into the actuators, these preliminary tests demonstrated the electroactivity of the high-aspect-ratio cylindrical ionogels. SCBD metallization of the micro-cylinders can be optimized by implementing several solutions. Masters with appropriate surface finishing 
and controlled roughness would favour the deposition and have potential to increase the electrodes' conductivity as well $[14,33]$. A more homogeneous electrical connection between the actuating body and basis interconnects of the ionogel could be obtained by identifying the critical tilting angles of the SCBD sample holder to effectively coat non-planar structures [27]. Moreover, the addition of suitable electrolytes and functional nanostructures into the ionogel matrix would be beneficial to further increase the material ionic conductivity and improve the actuation response in terms of displacement and response time [10].

The fabrication method proposed enables new applicative scenarios of soft devices with functional embodiments due to the synergy between replica moulding and SBCD. For example, cylindrical pillars can be used to form arrays of artificial electro-active cilia thanks to their slender geometry. Moreover, ionogel-based nanocomposites are not only interesting for their actuation characteristic, but also for their sensing ability. When an ionogel structure deforms, an electrical signal can be detected at the electrodes due to the variation of the ions concentrations inside the polymer matrix (piezo-ionic effect [12]). Therefore, this particular combination of geometry and materials can lead to a new generation of self-sensing actuators that open new horizons in small-scale soft robotics, from grippers with integrated haptic feedback to self-coordinated artificial cilia. The high electrochemical capacitance of the nanocomposites also has potential to be exploited for the design and manufacturing of energy storage functional devices with complex 3D architectures.

\section{Conclusions}

In this work, we report on the combination of replica molding and SCBD to fabricate high-aspect-ratio cylindrical structures of soft ionogel/gold nanocomposites. We used commercial metallic needles as masters to produce PDMS molds to fabricate ionogel-based cylinders $(0.5 \mathrm{~mm}$ diameter, aspect ratio $>20$ ). The cylinders are then metallized by means of SCBD to provide the polymer with integrated conductive gold nanostructure electrodes (150 nm thick). Nanocomposite thin films were used to assess the electrochemical and the electromechanical properties of the material, which revealed large electrochemical capacitance $\left(24 \mu \mathrm{F} / \mathrm{cm}^{2}\right)$ for charge storage and a moderate ionic conductivity of about $0.05 \mathrm{mS} / \mathrm{cm}$.

Actuation tests showed the electroactivity of the material in response to applied voltages ranging from $2.5 \mathrm{~V}$ to $5 \mathrm{~V}$ and a stable frequency response, while the actuation properties of the micro-cylinders were preliminarily investigated using remotely controlled electrodes. We foresee the implementation of such smart nanocomposite components for the realization of soft electroactive micro-systems with motion ability inspired by that of biological cilia.

Author Contributions: E.M. and T.S. conceived the research and wrote the manuscript; P.A. manufactured the structures and characterized the material; E.M., T.S. and L.M. critically analyzed the experimental results; P.M. supervised and supported the research. All authors revised the manuscript. All authors have read and agreed to the published version of the manuscript.

Funding: This research was supported by the Fund for Scientific Research-Flanders (FWO).

Conflicts of Interest: The authors declare no conflict of interest.

\section{References}

1. Rus, D.L.; Tolley, M.T. Design, fabrication and control of soft robots. Nature 2015, 521, 467-475. [CrossRef] [PubMed]

2. Hines, L.; Petersen, K.; Lum, G.Z.; Sitti, M. Soft Actuators for Small-Scale Robotics. Adv. Mater. 2016, 29. [CrossRef] [PubMed]

3. Carpi, F.; Kornbluh, R.; Sommer-Larsen, P.; Alici, G. Electroactive polymer actuators as artificial muscles: Are they ready for bioinspired applications? Bioinspiration Biomim. 2011, 6, 045006. [CrossRef] [PubMed]

4. Nardinocchi, P.; Pezzulla, M.; Placidi, L. Thermodynamically based multiphysic modeling of ionic polymer metal composites. J. Intell. Mater. Syst. Struct. 2011, 22, 1887-1897. [CrossRef] 
5. Bhandari, B.; Lee, G.-Y.; Ahn, S.-H. A review on IPMC material as actuators and sensors: Fabrications, characteristics and applications. Int. J. Precis. Eng. Manuf. 2012, 13, 141-163. [CrossRef]

6. Wang, J.; Xu, C.; Taya, M.; Kuga, Y. A Flemion-based actuator with ionic liquid as solvent. Smart Mater. Struct. 2007, 16, S214-S219. [CrossRef]

7. Pugal, D.; Jung, K.; Aabloo, A.; Kim, K.J. Ionic polymer-metal composite mechanoelectrical transduction: Review and perspectives. Polym. Int. 2010, 59, 279-289. [CrossRef]

8. Okazaki, H.; Sawada, S.; Kimura, M.; Tanaka, H.; Matsumoto, T.; Ohtake, T.; Inoue, S. Soft Actuator Using Ionic Polymer-Metal Composite Composed of Gold Electrodes Deposited Using Vacuum Evaporation. IEEE Electron Device Lett. 2012, 33, 1087-1089. [CrossRef]

9. Yan, Y.; Santaniello, T.; Bettini, L.G.; Minnai, C.; Bellacicca, A.; Porotti, R.; Denti, I.; Faraone, G.; Merlini, M.; Lenardi, C.; et al. Electroactive Ionic Soft Actuators with Monolithically Integrated Gold Nanocomposite Electrodes. Adv. Mater. 2017, 29. [CrossRef]

10. Santaniello, T.; Migliorini, L.; Yan, Y.; Lenardi, C.; Milani, P. Supersonic cluster beam fabrication of metal-ionogel nanocomposites for soft robotics. J. Nanoparticle Res. 2018, 20, 250. [CrossRef]

11. Milana, E.; Gorissen, B.; Peerlinck, S.; De Volder, M.; Reynaerts, D. Artificial Soft Cilia with Asymmetric Beating Patterns for Biomimetic Low-Reynolds-Number Fluid Propulsion. Adv. Funct. Mater. 2019, 29, 1-8. [CrossRef]

12. Villa, S.M.; Mazzola, V.M.; Santaniello, T.; Locatelli, E.; Maturi, M.; Migliorini, L.; Monaco, I.; Lenardi, C.; Franchini, M.C.; Milani, P. Soft Piezoionic/Piezoelectric Nanocomposites Based on Ionogel/BaTiO3 Nanoparticles for Low Frequency and Directional Discriminative Pressure Sensing. ACS Macro Lett. 2019, 8, 414-420. [CrossRef]

13. Johnston, I.D.; McCluskey, D.K.; Tan, C.K.L.; Tracey, M.C. Mechanical characterization of bulk Sylgard 184 for microfluidics and microengineering. J. Micromech. Microeng. 2014, 24, 035017. [CrossRef]

14. Corbelli, G.; Ghisleri, C.; Marelli, M.; Milani, P.; Ravagnan, L. Highly Deformable Nanostructured Elastomeric Electrodes With Improving Conductivity Upon Cyclical Stretching. Adv. Mater. 2011, 23, 4504-4508. [CrossRef]

15. Santaniello, T.; Migliorini, L.; Borghi, F.; Yan, Y.; Rondinini, S.; Lenardi, C.; Milani, P. Spring-like electroactive actuators based on paper/ionogel/metal nanocomposites. Smart Mater. Struct. 2018, 27, 065004. [CrossRef]

16. Migliorini, L.; Santaniello, T.; Rondinini, S.; Saettone, P.; Franchini, M.C.; Lenardi, C.; Milani, P. Bioplastic electromechanical actuators based on biodegradable poly(3-hydroxybutyrate) and cluster-assembled gold electrodes. Sens. Actuators B Chem. 2019, 286, 230-236. [CrossRef]

17. Wegner, K.; Piseri, P.; Tafreshi, H.V.; Milani, P. Cluster beam deposition: A tool for nanoscale science and technology. J. Phys. D Appl. Phys. 2006, 39, R439-R459. [CrossRef]

18. Piseri, P.; Tafreshi, H.V.; Milani, P. Manipulation of nanoparticles in supersonic beams for the production of nanostructured materials. Curr. Opin. Solid State Mater. Sci. 2004, 8, 195-202. [CrossRef]

19. Borghi, F.; Podestà, A.; Piazzoni, C.; Milani, P. Growth Mechanism of Cluster-Assembled Surfaces: From Submonolayer to Thin-Film Regime. Phys. Rev. Appl. 2018, 9, 044016. [CrossRef]

20. Mirigliano, M.; Borghi, F.; Podestá, A.; Antidormi, A.; Colombo, L.; Milani, P. Non-ohmic behavior and resistive switching of Au cluster-assembled films beyond the percolation threshold. Nanoscale Adv. 2019, 1, 3119-3130. [CrossRef]

21. Johnson, R.W.; Hultqvist, A.; Bent, S.F. A brief review of atomic layer deposition: From fundamentals to applications. Mater. Today 2014, 17, 236-246. [CrossRef]

22. Guo, H.C.; Ye, E.; Li, Z.; Han, M.-Y.; Loh, X.J. Recent progress of atomic layer deposition on polymeric materials. Mater. Sci. Eng. C 2017, 70, 1182-1191. [CrossRef] [PubMed]

23. Kusoglu, A.; Weber, A.Z. Electrochemical/Mechanical Coupling in Ion-Conducting Soft Matter. J. Phys. Chem. Lett. 2015, 6, 4547-4552. [CrossRef] [PubMed]

24. Ravagnan, L.; Divitini, G.; Rebasti, S.; Marelli, M.; Piseri, P.; Milani, P. Poly(methyl methacrylate)-palladium clusters nanocomposite formation by supersonic cluster beam deposition: A method for microstructured metallization of polymer surfaces. J. Phys. D Appl. Phys. 2009, 42, 082002. [CrossRef]

25. Cardia, R.; Melis, C.; Colombo, L. Neutral-cluster implantation in polymers by computer experiments. J. Appl. Phys. 2013, 113, 224307. [CrossRef]

26. Bellacicca, A.; Santaniello, T.; Milani, P. Embedding electronics in 3D printed structures by combining fused filament fabrication and supersonic cluster beam deposition. Addit. Manuf. 2018, 24, 60-66. [CrossRef] 
27. Santaniello, T.; Milani, P. Additive Nano-Manufacturing of 3D Printed Electronics Using Supersonic Cluster Beam Deposition. In Frontiers of Nanoscience; Elsevier: Amsterdam, The Netherlands, 2020; Volume 15, pp. 313-333.

28. Le Bideau, J.; Viau, L.; Vioux, A. Ionogels, ionic liquid based hybrid materials. Chem. Soc. Rev. 2011, 40, 907-925. [CrossRef]

29. Ding, Y.; Zhang, J.; Chang, L.; Zhang, X.; Liu, H.; Jiang, L. Preparation of High-Performance Ionogels with Excellent Transparency, Good Mechanical Strength, and High Conductivity. Adv. Mater. 2017, 29. [CrossRef]

30. Kim, K.J.; Tadokoro, S. Electroactive Polymers for Robotic Applications. Artif. Muscles Sens. 2007, 23, 291. [CrossRef]

31. Bian, C.; Zhu, Z.; Bai, W.; Chen, H.; Li, Y. Fast actuation properties of several typical IL-based ionic electro-active polymers under high impulse voltage. Smart Mater. Struct. 2020, 29, 035014. [CrossRef]

32. Aabloo, A.; Belikov, J.; Kaparin, V.; Kotta, U. Challenges and Perspectives in Control of Ionic Polymer-Metal Composite (IPMC) Actuators: A Survey. IEEE Access 2020, 8, 121059-121073. [CrossRef]

33. Marelli, M.; Divitini, G.; Collini, C.; Ravagnan, L.; Corbelli, G.; Ghisleri, C.; Gianfelice, A.; Lenardi, C.; Milani, P.; Lorenzelli, L. Flexible and biocompatible microelectrode arrays fabricated by supersonic cluster beam deposition on SU-8. J. Micromech. Microeng. 2011, 21, 45013. [CrossRef]

Publisher's Note: MDPI stays neutral with regard to jurisdictional claims in published maps and institutional affiliations.

(C) 2020 by the authors. Licensee MDPI, Basel, Switzerland. This article is an open access article distributed under the terms and conditions of the Creative Commons Attribution (CC BY) license (http://creativecommons.org/licenses/by/4.0/). 